\title{
Biceps Brachii Muscle Fatigue Assessment Through EMG Median Frequency Analysis
}

\author{
Ahmed Ebied \\ Biomedical engineering department, \\ Military Technical College, Cairo Egypt \\ E-mail: ahmed.m.ebied@gmail.com
}

\begin{abstract}
In this study, Surface electromyography (sEMG) signal is used to evaluate the biceps brachii muscle by estimating the muscle fatigue during isometric contraction. Sample of eight males performed isometric action using the biceps brachii muscle with different levels of force and loads. The surface electromyography (sEMG) signal was recorded by the MP 100 EMG system (BIOPAC Systems, California) for 120 seconds intervals without rest in order to achieve the fatigue, the recorded signals were analysed to find parameters in the sEMG that could estimate the muscle fatigue. The median frequency (MDF) of the power spectral density (PSD) and the root mean square value (RMS) were used as spectral and temporal variables to assess fatigue. Decreasing trend was found for (MDF) with time as the biceps muscle fatigues, while the (RMS) did not express consistent tendency with the fatigue process. Least square regression line model were adopted for each variable to form an index for fatigue assessment. The subjects were classified according to this index at the three loads to check the ability of the MDF to evaluate the muscular fatigue for each individual.
\end{abstract}

\section{I.INTRODUCTION}

There are many diverse and divergent descriptions and explanations for the word "fatigue". The issue of fatigue is complex due to the various physiological and psychological phenomena which contribute to it and which demonstrate it. In general, most currently used methods for measuring fatigue in the active human are doubly subjective, that is, they rely on the cooperation of the individual performing a prescribed task and the disposition of the observer when assessing the performed task. Therefore, it is common to think of an individual fatigues, when a particular task cannot be performed at a specific time. Such a notion of fatigue is inaccurate. For example, the task would be maintaining a muscle contraction at constant force for as long as possible. Throughout this task, the involved muscles are continuously fatiguing, but at one instant in time, the "failure point" will occur when the desired force output may no longer be maintained and contractile fatigue becomes observable. Therefore, it is more accurate to define fatigue as an exercise-induced decline in performance (1); it is task dependent and produces impairments either in the activation signal or muscle contractility (2) (3).

In other words, the fatigue is an underlying process which leads to the failure point where mechanical manifestations could be observed. During this process; strong modifications in the intra and extra-cellular environment arise (4), the electric properties of the muscular fibre membrane change (5), leading to myoelectric manifestations of muscle fatigue (2) (6) .Surface electromyography (sEMG) is a good indicator to the muscle performance as it measures the electrical activity of the muscle. 
The Surface electromyography (sEMG) signal considered as a weighted summation of the spatial and temporal activity of Motor Units Action Potentials (MUAPs) that propagate along the fibers of the desired muscle. Therefore; in order to evaluate muscular fatigue, sEMG is used as it is well known how myoelectric manifestations change according to fatigue type (7).

Muscle fatigue is strongly related to changes in frequency spectrum during various types of muscle contraction. (8) (9) (10). During continuous isometric contraction, the (sEMG) power spectral density (PSD) is observed to shift towards the lower frequencies. These spectral changes are generally attributed to a progressive decrease in muscle fiber conduction velocity (MFCV) (11), even though there are also other possible causes (12) (13) (14). In general, the observed shift of (PSD) to lower frequencies is mostly due to biochemical changes during fatiguing contraction in the type II muscle fibers (15). On the other hand; the amplitude of the sEMG during sustained muscle contraction increases due to the synchronization of the recruited motor units (MU) and the activation of new ones (13) (16); recruited MUs are becoming fatigued and new MU must be activated in order to sustain set levels of output (force, torque, power, speed).

Since the median frequency (MDF) is the frequency below which 50 percent of the signal power is found, it is a proper choice to quantify the PSD shift towards the lower frequency during the progression of muscle fatigue when a continuous isometric contraction of the muscle is performed (11). While the root mean square value (RMS) describes the amplitude variations by representing the mean power of the EMG signal.

This study is an approach for muscle assessment by investigating the fatigue process, in order to find indices that could characterize the fatigue properties of a specific muscle, which perform an isometric action under constant level of load for a certain time. This procedure will be discussed further in the next section followed by the results and discussion.

\section{A. Experimental Procedure}

\section{II.METHODS}

Eight healthy male subjects (average age $=22.8+/-2.1$ years) participated in this study (Table 1). All subjects were right handed and free from neurological dysfunction, neuropathy or myopathy. They were requested to stand with their right elbow flexed to a $90^{\circ}$ angle handling a 3.6, 4 and 6 kilograms load as their forearm positioned in horizontal position with hand palm up while recording the sEMG signal continuously for 120 seconds in order to reach fatigue (Figure 1)

Table 1 Anthropometric Characteristics of the participated subjects.

\begin{tabular}{|l|c|c|c|c|}
\hline Subjects & Age(year) & Body mass (Kg) & Height (cm) & Biceps diameter (cm) \\
\hline M1 & 21 & 69 & 172 & 31 \\
\hline M2 & 21 & 73 & 179 & 30 \\
\hline M3 & 21 & 70 & 180 & 39 \\
\hline M4 & 22 & 67 & 173 & 35 \\
\hline M5 & 26 & 80 & 190 & 31 \\
\hline M6 & 24 & 70 & 174 & 34 \\
\hline M7 & 26 & 79 & 174 & 33 \\
\hline M8 & 22 & 77 & & \\
\hline
\end{tabular}


In order to extract the sEMG (differential mode) in accord to the SENIAM Protocol (17), disposable, pre-gelled, (silver/silver chloride) pair of surface electrodes (with $1 \mathrm{~cm}$ apart) were placed over the longitudinal axis of the biceps brachii muscle parallel to the estimated direction of muscle fibers. Another disposable reference electrode was applied to the hand palm for artifacts $(<10 \mathrm{~Hz})$ limitation. Data were collected using the MP100 EMG system (BIOPAC Systems, California). The sEMG signal was band-pass filtered 1-5000 Hz and sampled at $10000 \mathrm{~Hz}$. The sEMG data were recorded and converted into ASCII format and saved into files for off-line analysis.

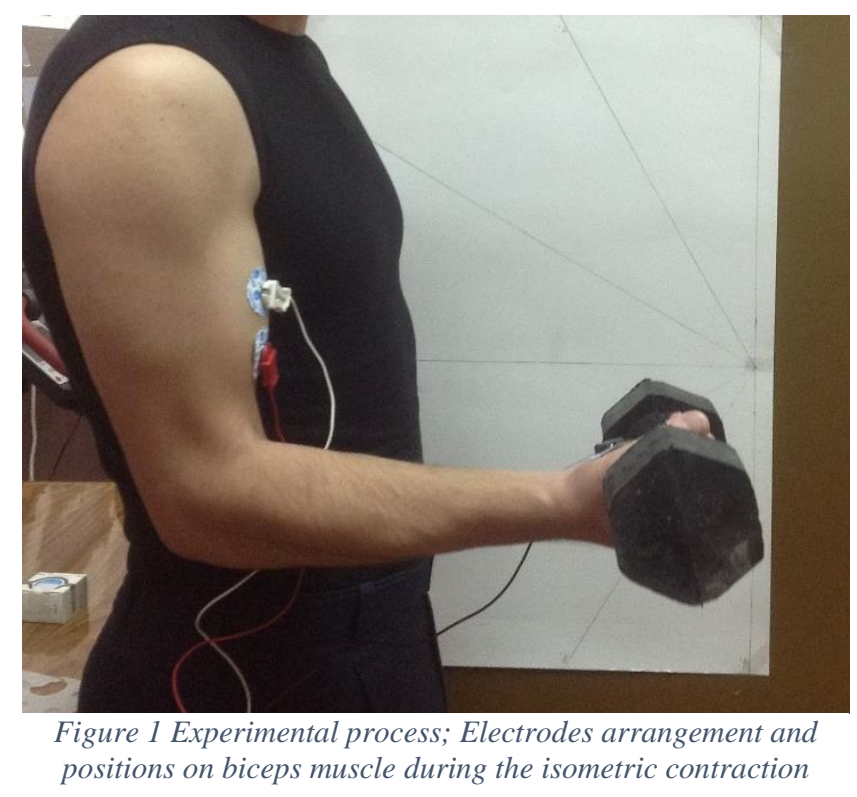

B. Data Analysis.

The MatLab software package (The Mathworks, Inc., MA) was used to analyze the sEMG, as the spectral and amplitude variables computed with numerical algorithm (time and frequency analysis). Prior to analysis, the sEMG signals were filtered with sixth-order Butterworth notch filter with quality factor of 50 in order to eliminate the power line noise, a bidirectional filtering technique was used disregard the phase shift. Then the signals were de-trended to eliminate the motion artifacts for better frequency analysis (Figure 2a). The shift in the PSD due to the fatigue could be recognized by comparing the PSD of the sEMG signal before and after the fatigue. In other words, at the beginning and at the end of the recorded sEMG. (Figure 2c).

In order to, assess the time related variations in the spectral and amplitude variables (MDF and RMS), the sEMG signals were segmented into 1 second epochs where the Root Mean Square value computed in addition to Fast Fourier Transform (FFT) calculation for Median frequency computation. The data length of $1000 \mathrm{~ms}$ was chosen as the optimum value based on the literature (18) and (19) where a comparison was held between data window lengths between 125 to $1000 \mathrm{~ms}$ on data recorded during prolonged isometric contraction and found no significant effect on frequency parameters under investigation. 
Moreover, both variables (RMS and MDF) were plotted as functions of time with a 1-second increment. Using visual and graphical inspections these variables showed linear behavior. Thus, a linear model $\left(y=b_{1} x+\right.$ $b_{0}$ ) is adopted for each variable, to perform statistical processing. A least squares regression line was fitted to each data set. The slope of the linear model of the MDF variable $\left(b_{1}\right)$ is then considered as an index of fatigue rate whereas the intercept $\left(b_{0}\right)$, indicates the initial value of the outcome variable. In similar way the RMS parameters were fitted with linear model $y=a_{1} x+a_{0}$ to investigate any relation between fatigue and the time domain parameters.

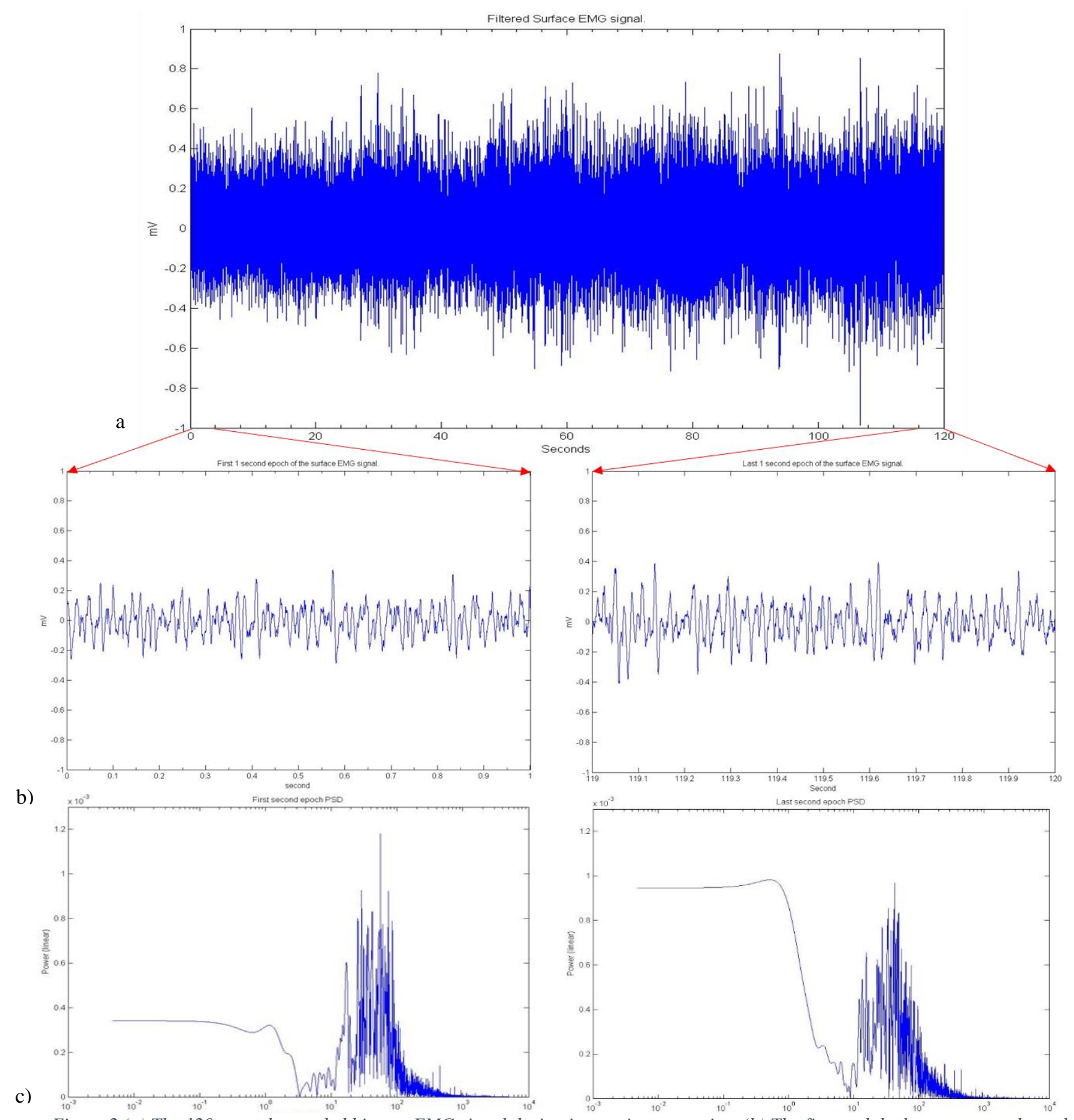

Figure 2 (a) The 120 second recorded biceps sEMG signal during isometric contraction. (b) The first and the last one second epochs in time domain. (c) The PSD of the first and the last epoch. 


\section{III.RESULTS AND DISSCUSION}

All subjects completed the protocol successfully under three different loads with 5 minutes rest in between. The MDF and RMS were calculated from each 1 second epoch in the total 120 second sEMG signal. The median frequency variable showed consistent linear to moderately curvilinear behavior as a function of time for each subject at all loads. The slope $\left(b_{1}\right)$ and the intercept $\left(b_{0}\right)$ were calculated from the least regression model for each sEMG signal of 120 seconds of sustained isometric contraction of the biceps brachii. As an illustration, the MDF values for each 1 second epoch plotted as a function of time with their least squares regression line presented in. (Figure 3).

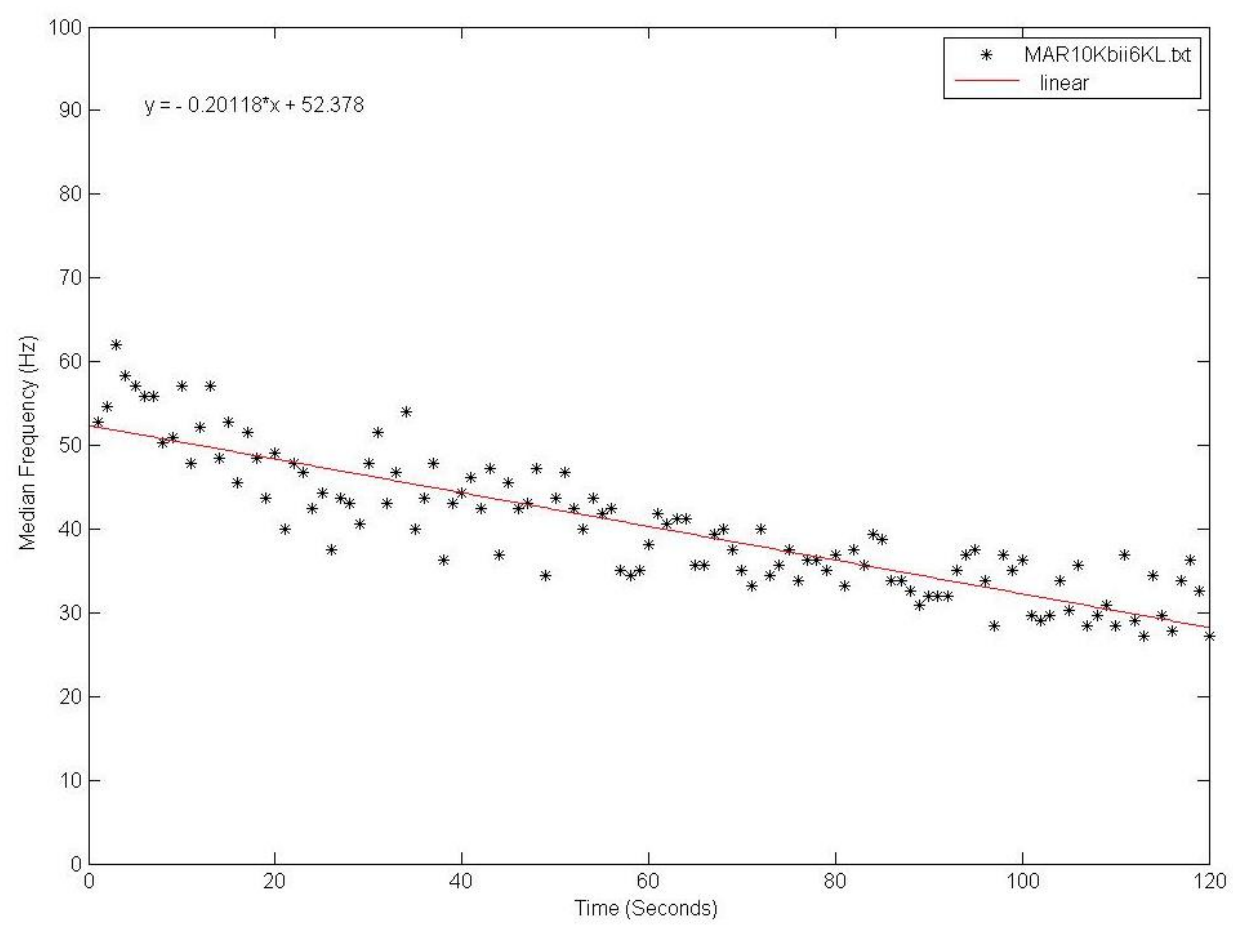

Figure 3 Sample plot of the median frequency (MDF) of surface EMG detected from biceps brachii during 120 seconds of isometric contraction. Regression line is shown with its linear model where $b 1=-0.020118 \mathrm{~Hz} / \mathrm{sec}$ and $b 0=52.378 \mathrm{~Hz}$

The arithmetic mean of the Median Frequency (MDF) slopes $\left(b_{1}\right)$ for all subjects were $(-0.0629972$ and $0.068221 \mathrm{~Hz} / \mathrm{s})$ under $3.6 \mathrm{Kg}$ load and $4 \mathrm{Kg}$ load respectively while it was higher at $6 \mathrm{Kg}$ load $(-0.09829 \mathrm{~Hz} / \mathrm{s})$. On the other hand the MDF intercept $\left(\mathrm{b}_{0}\right)$ were comparable under all loads as same as the RMS parameters $\left(a_{1}\right)$ and $\left(a_{0}\right)$ which did not show a consistent patterns.

The eight subjects were classified according to the value of MDF slope $\left(b_{1}\right)$ at the three different levels of loads to investigate if this parameter could be used as an indication of the rate of fatigue in order to evaluate the muscle performance. In addition to the MDF slope parameter, all other parameters are summarized in three groups in (Table 2), (Table 3) and. (Table 4) according to load level $6 \mathrm{Kg}$, and $4 \mathrm{Kg}$ and $3.6 \mathrm{Kg}$ respectively. 
Table 2 MDF and RMS intercept and slope of the regression lines for all subjects during the 120 second isometric contraction at 6 Kg load arranged according to $b_{1}$

\begin{tabular}{|l|l|l|l|l|}
\hline $\mathbf{6}$ Kg Load & $\boldsymbol{b}_{\mathbf{0}}$ & $\boldsymbol{b}_{\mathbf{1}}$ & $\boldsymbol{a}_{\mathbf{0}}$ & $\boldsymbol{a}_{\mathbf{1}}$ \\
\hline M1 & 52.378 & -0.20118 & 0.23838 & 0.0036379 \\
\hline M2 & 58.561 & -0.11926 & 0.11198 & 0.0000186 \\
\hline M3 & 50.605 & -0.088719 & 0.17134 & 0.0017112 \\
\hline M4 & 56.946 & -0.088613 & 0.18479 & 0.0005419 \\
\hline M5 & 56.534 & -0.079779 & 0.16311 & -0.000024 \\
\hline M6 & 45.407 & -0.077213 & 0.12805 & 0.0005762 \\
\hline M7 & 49.332 & -0.07113 & 0.11394 & 0.0005862 \\
\hline M8 & 56.09 & -0.060427 & 0.16027 & 0.0005472 \\
\hline
\end{tabular}

Table 3 MDF and RMS intercept and slope of the regression lines for all subjects during the 120 second isometric contraction at 4 Kg load arranged according to $b_{1}$. M3 seem to have irregular $b_{1}$ value in addition to the greatest al value.

\begin{tabular}{|l|l|l|l|l|}
\hline 4 Kg Load & $\boldsymbol{b}_{\mathbf{0}}$ & $\boldsymbol{b}_{\mathbf{1}}$ & \multicolumn{1}{|c|}{$\boldsymbol{a}_{\mathbf{0}}$} & $\boldsymbol{a}_{\mathbf{1}}$ \\
\hline M1 & 50.954 & -0.18163 & 0.13118 & 0.0004141 \\
\hline M2 & 58.256 & -0.072773 & 0.095318 & -0.000052 \\
\hline M4 & 53.107 & -0.067533 & 0.10567 & 0.0001206 \\
\hline M6 & 52.171 & -0.029697 & 0.10759 & -0.000088 \\
\hline M5 & 46.475 & -0.029545 & 0.09532 & 0.0000167 \\
\hline M3 & 45.755 & -0.028148 & 0.12944 & 0.0039584 \\
\hline M7 & 53.975 & -0.027739 & 0.11123 & 0.0000102 \\
\hline M8 & 41.855 & -0.002007 & 0.076738 & 0.0002446 \\
\hline
\end{tabular}

Table 4MDF and RMS intercept and slope of the regression lines for all subjects during the 120 second isometric contraction at $3.6 \mathrm{Kg}$ load arranged according to bI.M5, M6 and M7 had very close bivalues at the three loads.

\begin{tabular}{|l|l|l|l|l|}
\hline 3.6 Kg Load & $\boldsymbol{b}_{\mathbf{0}}$ & $\boldsymbol{b}_{\mathbf{1}}$ & $\boldsymbol{a}_{\mathbf{0}}$ & \multicolumn{1}{|c|}{$\boldsymbol{a}_{\mathbf{1}}$} \\
\hline M1 & 78.593 & -0.10862 & 0.48282 & 0.0012258 \\
\hline M2 & 65.447 & -0.079065 & 0.30525 & 0.000231 \\
\hline M3 & 52.812 & -0.049756 & 0.39037 & 0.00050839 \\
\hline M4 & 54.835 & -0.028928 & 0.34872 & 0.0016791 \\
\hline M6 & 56.271 & -0.024451 & 0.06789 & 0.000643 \\
\hline M5 & 53.699 & -0.024241 & 0.07462 & 0.000236 \\
\hline M7 & 54.107 & -0.022055 & 0.051772 & -0.000066 \\
\hline M8 & 52.519 & -0.019308 & 0.032155 & 0.0001082 \\
\hline
\end{tabular}


Comparing the eight subject performance during the three different loads according to $\left(b_{1}\right)$ shows a regular trend (Figure 4). The rate of fatigue - which could be represented as the absolute value of $\left(b_{1}\right)$ - for each individual is relatively the same in the three different loads in comparison to other individuals except for M3. The value of $\left(b_{1}\right)$ or the rate of fatigue for M3 at $4 \mathrm{Kg}$ load is less than anticipated according to the other two values from the other two loads. Moreover, the subjects M5, M6 and M7 shows approximately similar results with very close values at all loads.

It is noticed that the greatest value of RMS slope $\left(a_{1}\right)$ is associated to the M3 recorded surface EMG signal with applied load of $4 \mathrm{Kg}$. Therefore, a relation may be exist between $\left(\mathrm{a}_{1}\right)$ parameter and the reliability of the recorded signal as the applied voluntary force may vary under constant load.

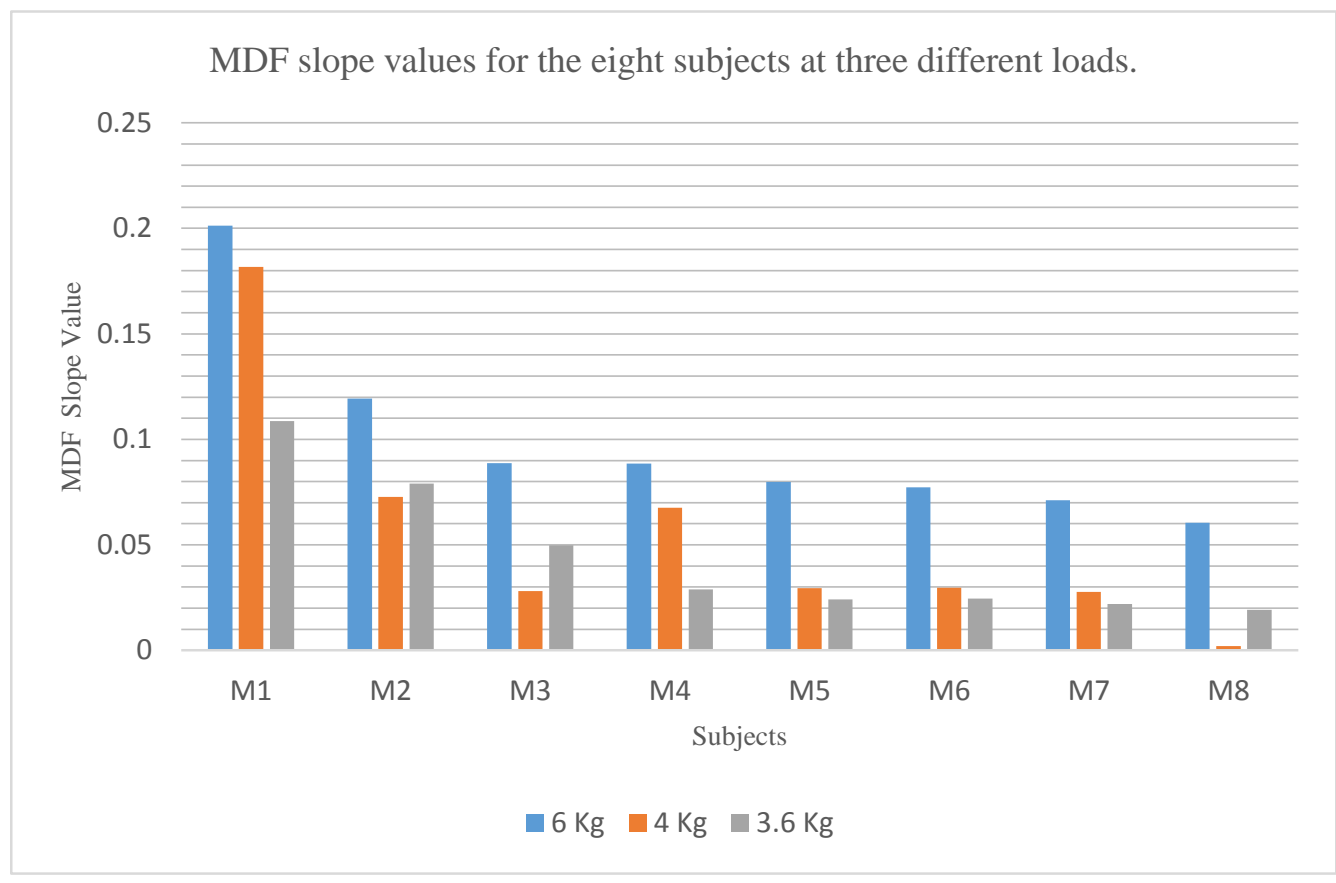

Figure 4 Comparison between the eight subjects at the three loads according to the MDF slope absolute value which could be considered as an index of fatigue.

According to those results, the change in median frequency of a surface EMG recorded through a sustained isometric contraction is a good indication of muscle fatigue. Furthermore, this rate of change could be used as an index of the rate of muscle fatigue in order to characterize the muscle to evaluate the physical performance of an individual.

\section{IV.CONCLUSION}

This study introduces the median frequency as a tool to evaluate the performance of the skeletal muscle from the rate of fatigue point of view. The rate of fatigue was measured at three levels of different load. It was observed that the rate of fatigue increases with the load and applied force, although, the rate of fatigue persist relatively the same for different individual. The median frequency index was able to discriminate between subjects according to their rate of fatigue despite of some of the results that were very close to each other.

One of the results showed irregular trend in the rate of fatigue index and was characterized with the highest RMS change value which may indicate a possible inaccuracy in the recorded surface EMG signal. 
To sum up, the MDF index provides a suitable basis for developing an evaluation model to assess the muscle's level of fatigue

\section{V.REFERENCES}

1. Effect of muscle fatigue on the sense of limb position and movement. Allen TJ, Proske U. 1, s.l. : Experimental Brain Research, 2006, Vol. 170.

2. Myoelectric manifestations of fatigue in voluntary and electrically elicited contractions. Merletti R, Knaflitz M, De Luca CJ. 5, s.1. : Journal of Applied Physiology, 1990, Vol. 69.

3. Electrical manifestations of muscle fatigue during concentric and eccentric isokinetic knee flexion-extension movements. Molinari

F, Knaflitz M, Bonato P, Actis MV. 7, s.1. : Biomedical Engineering, IEEE Transactions on, 2006, Vol. 53.

4. Interpretation of EMG changes with fatigue: facts, pitfalls, and fallacies. Dimitrova NA, Dimitrov GV. 1, s.1.: Journal of Electromyography and Kinesiology, 2003, Vol. 13.

5. The change in spatial distribution of upper trapezius muscle activity is correlated to contraction duration. Farina $\mathbf{D}$, Leclerc $\mathbf{F}$, Arendt-Nielsen L, Buttelli O, Madeleine P. 1, s.1. : Journal of Electromyography and Kinesiology, 2008 , Vol. 18.

6. The use of surface electromyography in biomechanics. CJ., De Luca. 1, s.1. : JOURNAL OF APPLIED BIOMECHANICS, 1997, Vol. 13.

7. John V. Basmajian, Carlo J. De Luca. Muscle alive. s.l. : Williams \& Wilkins, 1985.

8. Changes in muscle fiber conduction velocity, mean power frequency, and mean EMG voltage during prolonged submaximal contractions. Arendt-Nielsen L, Mills KR, Forster A. 6, s.1. : Muscle Nerve, 1989, Vol. 12, pp. 493-7.

9. EMG power spectrum of respiratory and skeletal muscles during static contraction in healthy man. Badier M, Guillot C, LagierTessonnier F, Burnet H, Jammes Y. 6, s.1. : Muscle Nerve, 1993, Vol. 16, pp. 601-9.

10. Myoelectrical manifestations of localized muscular fatigue in humans. CJ., De Luca. 4, s.l. : Critical Reviews in Biomedical Engineering, 1984, Vol. 11, pp. 251-79.

11. Conduction velocity in ischemic muscle: effect on EMG frequency spectrum. Mortimer JT, Magnusson R, Petersén I. 5, s.l. : American Journal of Physiology, 1970, Vol. 219, pp. 1324-9.

12. Myoelectric and mechanical manifestations of muscle fatigue in voluntary contractions. Merletti, R., Roy, S. 1, s.l. : Journal of Orthopedy and Sports Physical Therapy, 1996, Vol. 24, pp. 342-53.

13. Electromyogram median frequency, spectral compression and muscle fibre conduction velocity during sustained sub-maximal contraction of the brachioradialis muscle. Lowery M, Nolan P, O'Malley M. 2, s.l. : Journal of Electromyography and Kinesiology, 2002, Vol. 12, pp. 111-8.

14. Changes in surface EMG parameters during static and dynamic fatiguing contractions. Masuda, K., Masuda, T., Sadoyama, T., Inaki, M. \& Katsuta, S. 1, s.1. : Journal of Electromyography in Kinesiology, Vol. 9, pp. 39-46.

15. The relationships between EMG and muscle morphology throughout sustained static knee extension at two submaximal force levels. Gerdle B, Karlsson S, Crenshaw AG, Fridén J. 4, s.1. : Acta Physiologica Scandinavica, 1997, Vol. 160, pp. 341-51.

16. Merletti R, Rainoldi A, Farina D. Myoelectric Manifestations of Muscle Fatigue. Electromyography: Physiology, Engineering, and Noninvasive Applications. Hoboken : John Wiley \& Sons, Inc., 2004, 9.

17. Development of recommendations for SEMG sensors and sensor placement procedures. Hermens HJ, Freriks B, DisslhorstKlug C, Rau G. s.1. : Electromyogr Kinesiol, 2000, Vol. 10, pp. 361-74.

18. Comparison of algorithms for estimation of EMG variables during voluntary. Roberto Merletti, Farina. 10, s.1. : Journal of Electromyography and Kinesiology, 2000, Vol. 2, pp. 337-249.

19. Muscular fatigue during $200 \mathrm{~m}$ front crawl. Pedro Figueiredo, Suzana Pereira, Pedro Gonçalves,Ricardo J Fernandes. 11, s.1. : Portuguese Journal of Sport Sciences, 2011, Vol. 2, pp. 735-738. 